\title{
Evaluation and optimization of surveillance systems for rare and emerging infectious diseases
}

\author{
Daniela C. HADORN ${ }^{1 *}$, Katharina D.C. STÄRK ${ }^{1,2}$ \\ ${ }^{1}$ Federal Veterinary Office, Berne, Switzerland \\ ${ }^{2}$ Royal Veterinary College, London, United Kingdom
}

(Received 1 February 2008; accepted 23 July 2008)

\begin{abstract}
Surveillance for rare and emerging infectious diseases poses a special challenge to veterinary services. Most emerging infectious diseases like bovine tuberculosis (bTB) are zoonoses, affecting both human and animal populations. Despite the low prevalence of such an emerging infectious disease at time of incursion, the surveillance system should be able to detect the presence of the disease as early as possible. Because passive surveillance is a relatively cost-effective and therefore commonly used process, it is the basic tool for infectious disease surveillance. Because of under-reporting in passive surveillance, cost-intensive active surveillance is often required to increase the sensitivity of the surveillance system. Using scenario tree modelling, the sensitivity of passive and active surveillance system components (SSC) can be quantified and an optimal, cost-effective surveillance system developed considering the contributions of each SSC. We illustrate this approach with the example of bTB surveillance in Switzerland where the surveillance system for bTB consists of meat inspection at the slaughterhouse (SLI), passive clinical surveillance on farm (CLIN) and human surveillance (HS). While the sensitivities for CLIN and HS were both negligible $(<1 \%)$, SLI was assessed to be $55.6 \%$. The scenario tree model showed that SLI is increasable up to $80.4 \%$ when the disease awareness of meat inspectors in Switzerland is enhanced. A hypothetical random survey (RS) was also compared with a targeted survey (TS) in high-risk strata of the cattle population, and the sensitivity of TS was 1.17 -fold better than in RS but with $50 \%$ of the sample size.
\end{abstract}

scenario tree modelling / surveillance system / emerging infectious disease / bovine tuberculosis / Switzerland

\section{INTRODUCTION}

Over the last decade, surveillance for zoonotic pathogens has become more and more relevant in relation to emerging infectious diseases of animal origin. Such diseases pose a special challenge to veterinary authorities because they are initially rare and therefore difficult to detect. Most of the emerging infectious diseases are zoonotic diseases [15] A central characteristic of zoonoses in the context of animal surveillance is that veterinary services are confronted not only with animal but also with public health issues. Because of such expanding duties but generally

*Corresponding author: daniela.hadorn@bvet. admin.ch decreasing resources for animal health surveillance, current surveillance strategies need to be evaluated and optimized in terms of surveillance performance in relation to costs ${ }^{1}$.

In the context of emerging infectious diseases, the objective of surveillance is early detection when the incidence of cases is still low in order to allow rapid response. The most critical factor for early detection is the sensitivity of the surveillance system, i.e. the ability to detect an outbreak as soon as

\footnotetext{
${ }^{1}$ Within the scope of this paper, costs are only related to the required sample size for a survey to achieve a certain sensitivity. But it is obvious that there exist a lot of other cost categories that should be integrated in a further study.
} 
possible. According to the World Animal Health Organization (Office International des Epizooties, OIE), a surveillance system is a method of surveillance that may include one or more component activities that generates information on the health, disease or zoonosis status of animal populations ${ }^{2}$. The power of a surveillance system to detect cases can also be referred to as its sensitivity and can be estimated when the sensitivities of the surveillance system components are known. Surveillance system components (SSC) may be based on two different surveillance approaches, namely active and passive surveillance ${ }^{3}$ :

Active surveillance is defined as the periodic collection of samples or case reports from veterinary health authorities $[6,12]$. For example, conducting a diagnostic survey is an active surveillance approach to obtain valid information on the disease or infection status of a population at a given time. But this approach is cost-intensive, especially for rare diseases where a large sample size is required, due to the low expected prevalence. One way of making this approach more efficient is the application of riskbased surveillance by targeting the sampling on high-risk populations in which specific, documented risk factors exist and where the probability of finding cases is highest [16]. Thus, resources can be focused on population strata where the disease to be detected is anticipated to be more common than in a randomly chosen part of the population.

Passive surveillance is defined as the reporting of clinical suspect cases to the health authorities [12]. It is called 'passive' because the decision to test an animal or a sample is made by the animal owner, practitioner or meat inspector and not by the veterinary authority [6]. The sensitivity of passive surveillance depends on many factors

${ }^{2}$ OIE, Terrestrial Animal Health Code of the OIE. [online] (2007) http://www.oie.int/eng/normes/ Mcode/en_sommaire.htm [consulted 01/11/2007].

${ }^{3}$ The concepts of active and passive surveillance apply to both human and animal surveillance. But in this paper, the definitions refer to the surveillance of animal populations only.

Page 2 of 12 (page number not for citation purpose) including the probability of infected animals showing detectable clinical signs, the disease awareness of farmers, veterinarians and health authorities and their motivation to report, as well as the sensitivity of the confirmatory test. Therefore, it is difficult to quantify the sensitivity of passive surveillance and to assess its performance with regard to disease detection.

Although the sensitivity of passive surveillance is rarely quantified, it is usually assumed to be too biased to provide reliable information on the actual infection status of a population. Therefore, additional active surveillance is often required. Active as well as passive surveillance can be combined in one surveillance system for a specific animal disease or infection and are therefore SSC that contribute evidence to the disease or infection status of a population.

In this paper, we describe an approach for the quantification and evaluation of active and passive SSC and for the optimization of surveillance systems using scenario tree modelling. We illustrate our approach with the example of bovine tuberculosis (bTB) surveillance in Switzerland because bTB is classified by the World Health Organization ${ }^{4}$ as a neglected zoonosis and has the potential to re-emerge in Switzerland. It is also a major economic problem and a threat to public health in numerous countries [17].

\section{MATERIALS AND METHODS}

\subsection{Quantification of active and passive SSC}

To initiate the evaluation and optimization of a surveillance system, all SSC need to be identified and described. With a scenario tree approach, each element from the occurrence of an infection to the detection of the case is represented as a set of events with specified probabilities, and the overall probability, i.e. sensitivity to detect at least one

\footnotetext{
${ }^{4}$ World Health Organization, The control of neglected zoonotic diseases; a route to poverty alleviation. Report of a joint WHO/DFIDAHP meeting, 20 and 21 September 2005, in: Zoonoses and veterinary public health, [online] http://www.who.int/zoonoses/Report_Sept06.pdf [consulted 08/10/2007].
} 
positive unit given that the population is truly infected can be calculated for each individual SSC. For the implementation of this approach, we used the stochastic scenario tree approach which Martin et al. [13] originally developed to demonstrate freedom from infection using multiple complex data sources. This method incorporates the impact of population strata on case occurrence in a country or region by implementing relative risks (RR) for different sub-strata of the population. The RR are weighted according to the proportion of the SSC reference population so that the average adjusted risk (AR) for the population strata is one, as described by Martin et al. [13]:

$$
\sum_{l=1}^{L}\left(A R_{l} \times \operatorname{Pr} P_{l}\right)=1
$$

where $L$ is the number of strata ${ }^{5}$ for a specific risk factor and $\mathrm{PrP}_{l}$ is the proportion of units (= farms) that falls into the $l$ th stratum.

The sensitivity of a SSC (CSe) depends on the level of disease in the population and is called design prevalence $(\mathrm{P} * \mathrm{H})$ [13]. To take into account clustering of cases in high-risk population strata and to quantify CSe for individual risk region or population strata, the effective probability of infection (EPI) per risk stratum is calculated multiplying $\mathrm{P} * \mathrm{H}$ with any applicable AR [13]. In order to quantify $\mathrm{CSe}$, the probability that any randomly chosen farm out of the population will give a positive outcome (CSeU) has to be calculated first. This is done by multiplying the proportions of the farms falling into each of the specified population strata by the corresponding EPI as well as by the probabilities for detection. Then, the probabilities for all positive outcomes are summed up. Finally, CSe is calculated as follows [13]:

$$
\mathrm{CSe}=1-(1-\mathrm{CSeU})^{n}
$$

where $n$ is the number of processed farms.

Under the assumption that all SSC of a surveillance system are independent, CSe can be combined to an overall sensitivity of the surveillance system (SSe) using the following equation as described by Martin et al. [13]:

$$
S S e=1-\prod_{j=1}^{J}\left(1-C S e_{j}\right)
$$

\footnotetext{
${ }^{5}$ The number of strata used in this paper corresponds to the number of branches per node used in Martin et al. [13].
}

where $J$ is the number of surveillance system components in the country and $\mathrm{CSe}_{j}$ is the SSC sensitivity for the $j$-th component. Using this equation, the sensitivities of active as well as passive SSC can be taken into account for the overall assessment of the quality of a surveillance system.

\subsection{Evaluation and optimization of a surveillance system}

Once the sensitivities of the SSC are estimated, alternative designs can be explored to optimize the surveillance system in terms of cost-benefit ratio.

\subsubsection{Identification of risk factors and high-risk population strata}

Infectious disease is rarely homogeneously distributed within a population of susceptible livestock due to husbandry systems and possible climatic or geographical influence. Taking this clustering into account in the design of active SSC (surveys) leads to so-called targeted sampling (TS). All factors influencing the probability that a herd/flock or a single animal gets infected with a specific disease agent need to be identified using epidemiological studies. Based on the occurrence of such risk factors, the population can be divided into sub-strata with different risks of infection. Taking RR into account, EPI for each sub-stratum can be calculated as described before. Consequently, the occurrence of disease in an infected region or country is expected to be higher in 'high-risk' strata than in the other strata. Therefore in this work, the stochastic scenario tree model was also used for a comparison of random sampling (RS) with TS in defined high-risk population strata.

The sensitivity of RS and TS is dependent on the proportion of sampled farms per stratum $\left(\mathrm{Pr} \mathrm{P}_{l}\right)$ and its corresponding prevalence (EPI), the sample size ( $n$ ) and the test sensitivity. For RS, the distribution of sampled farms among the population strata is assumed to be congruent with the SSC reference population. This stands in contrast to TS where only farms out of specific risk strata are tested. Consequently, the proportion of all farms sampled that are in the high risk stratum is $100 \%$.

The probability that infected herds and animals will be detected may also be variable according to other factors, e.g. age of the animal or management system. Such risk factors for detection can equally be integrated in the analysis of the different SSC. In summary, all risk factors for infection and detection 
need to be identified in order to apply the approach proposed here. This is typically achieved by a systematic literature review and synthesis of the findings. From this follows that TS is of limited value when risk factors for a disease are unknown.

\subsubsection{Evaluation of active and passive SSC with regard to the overall surveillance system sensitivity}

In establishing an optimal surveillance system for emerging infectious diseases in terms of costbenefit ratio, the contribution of passive SSC to the surveillance system sensitivity deserves special attention. Using sensitivity analysis, factors can be identified which are most influential on the sensitivity of the SSC in the scenario tree models. Factors which can be influenced by interventions, e.g. disease awareness of farmers and practitioners, are a possible starting point for an economical increase in surveillance system sensitivity.

In a situation where increase in disease awareness through campaigns is not efficient enough or is impracticable, or for diseases without typical clinical symptoms, active SSC may need to be added to increase the sensitivity of the surveillance system. If risk factors and corresponding high-risk groups can be identified for the disease, TS is a cost-effective alternative to RS.

\subsection{Example of bTB surveillance in Switzerland}

\subsubsection{Identification of risk factors and high-risk population strata for bTB in Switzerland}

Switzerland has been officially free from bTB since 1959 [1]; i.e. the overall herd prevalence of bTB in Switzerland is $<0.2 \%^{2}$. However, three possible pathways for bTB introduction into Swiss cattle farms exist: (A) via infected wildlife; (B) via traded cattle; and (C) via bTB-infected humans working on cattle farms (human-to-cattle transmission). Exposure to each of these pathways therefore constitutes a risk factor for exposure to bTB.

(A) There is no established wildlife reservoir known for bTB in Switzerland ${ }^{6}$. In Italy, in

${ }^{6}$ Leuenberger R., Surveillance of wild boar in Switzerland: prevalence of infections relevant to domestic pigs, Philosophische-naturwissenschaftliche Fakultät der Universität Basel thesis, Basel, Switzerland, 2004.

Page 4 of 12 (page number not for citation purpose)
Lombardy region, some cases of bTB have been detected in wild boars [7]. Therefore, cattle in the cantons of Ticino and Grisons ${ }^{7}$ were defined as a high-risk population stratum labelled 'wildlife' and $6.5 \%$ of all cattle farms belong to this stratum (Fig. 1).

(B) According to Johnston et al. [10], movement of cattle onto the farm from markets or farm sales is one of the strongest factors associated with an increased bTB risk. In our model, cattle dealers (1.7\% of cattle farms) represent farms with high animal movement and are categorized in the highrisk stratum labelled 'trade'.

(C) bTB is a zoonosis and human-to-cattle transmission has occurred in the past also in Switzerland $[8,9]$. However, because bTB was eradicated from Switzerland almost 50 years ago, we assumed that the risk of human-tocattle transmission would most likely originate from foreign farm assistants working on cattle farms. Approximately 2000 foreign farm assistants originating primarily from Eastern Europe, where bTB may be present [14], are working on Swiss farms every year. Under the assumption that each farm assistant worked on one cattle farm, 3.3\% of Swiss cattle farms may be subject to human-tocattle transmission and this stratum was labelled 'human'.

In order to estimate RR for 'wildlife' $(\mathrm{RR}=5)$ and 'trade' $(R R=10)$, two bTB experts from UK were consulted and their relative risk data were adapted to Swiss situation in discussion with the authors. RR for 'human' was assumed to be 1.5 after consultation with a Swiss public health specialist.

\subsubsection{Evaluation of active and passive SSC for bTB surveillance}

\subsubsection{Passive SSC}

For the surveillance of bTB in Switzerland, the following SSC were considered: clinical surveillance on farm (CLIN); meat inspection at the slaughterhouse (SLI); and human clinical case surveillance (HS). CLIN describes the process of an infected cattle farm being detected through animal caretakers and practitioners. Given that a cattle farm is infected and that the disease produces clinical signs in infected animals, the case detection in

\footnotetext{
${ }^{7}$ The cantons of Ticino and Grisons are adjacent to Lombardy region.
} 


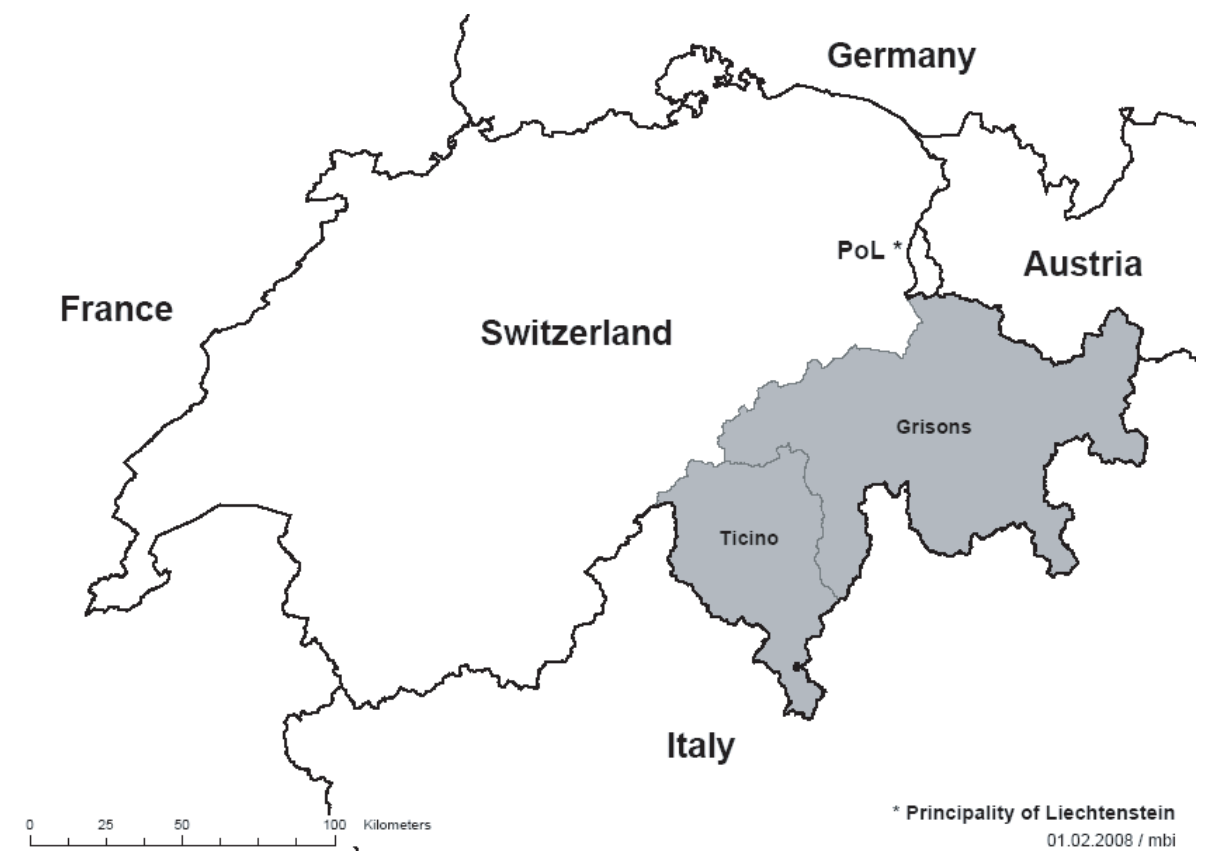

Figure 1. Map of Switzerland with the adjacent countries.

the CLIN-SSC depends on the disease awareness of the farmer and the veterinarian and on the tuberculin test sensitivity (Fig. 2a). The key SSC for bovine tuberculosis consists of the detection of visible lesions (VL) in the slaughterhouse (SLI) and further diagnostic procedures initiated by the meat inspector (Fig. 2b). Given that an infected animal shows visible lesions detectable post mortem, the awareness and the accuracy of the meat inspector is a crucial point for the sensitivity of this SSC $[2,11]$. Because bTB is a zoonosis and can be transmitted from cattle to people, human Mycobacterium bovis cases are indications of the presence of infected cattle. Therefore, each human bTB case should trigger a follow-up to rule out bTB in cattle (Fig. 3). The crucial points in this passive SSC based on human surveillance (HS) are the disease awareness of medical practitioners and the reporting of human bTB cases to the State Veterinary Service in order to conduct follow-up examinations of suspect cattle farms.

Disease awareness is difficult to quantify because it is influenced by different circumstances. Within the scope of this work, we assessed the disease awareness in Switzerland qualitatively using expert opinion and then transformed the qualitative ranges 'low', 'medium', 'high' and 'very high' into the corresponding quantitative input parameter shown in Table I. Using this classification, we calculated the sensitivity for each passive SSC for the assumed current Swiss situation (scenario 1, Tab. II) and compared this situation to three different hypothetical scenarios with each scenario having a different level of disease awareness (Tab. II).

In our evaluation, the evidence provided by passive SSC was analyzed over one year because bTB is a slow-moving disease ${ }^{8}$. The input parameters are shown in Table III. The stochastic simulation model was run with 10000 iterations 9 .

\subsubsection{Active SSC}

In Switzerland, the last random survey to document freedom from bTB was conducted in

\footnotetext{
${ }^{8}$ For more details on the implication of time period, we refer to Martin et al. [13].

${ }^{9}$ In this work, we used Microsoft Excel and Palisade@RISK [online](Palisade Corporation: www.palisade.com) as the modelling software [consulted 04/06/2007].
} 
a

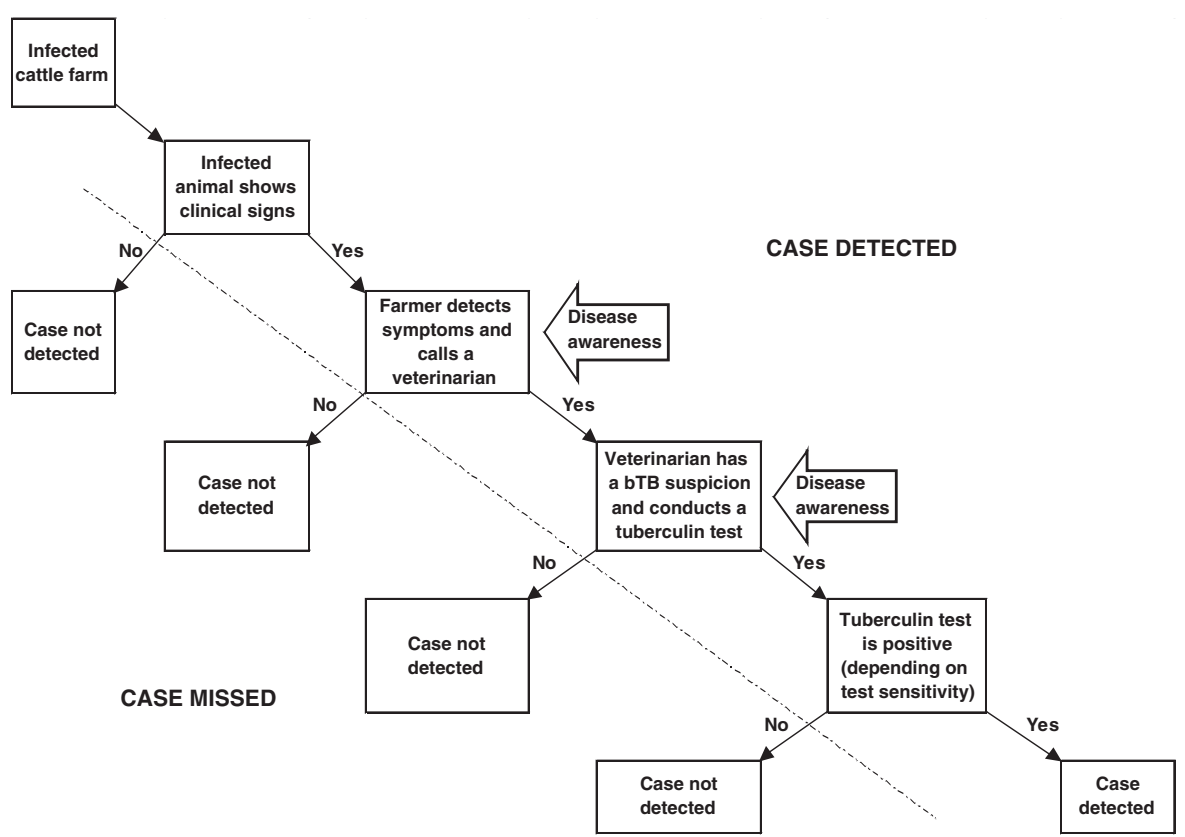

b

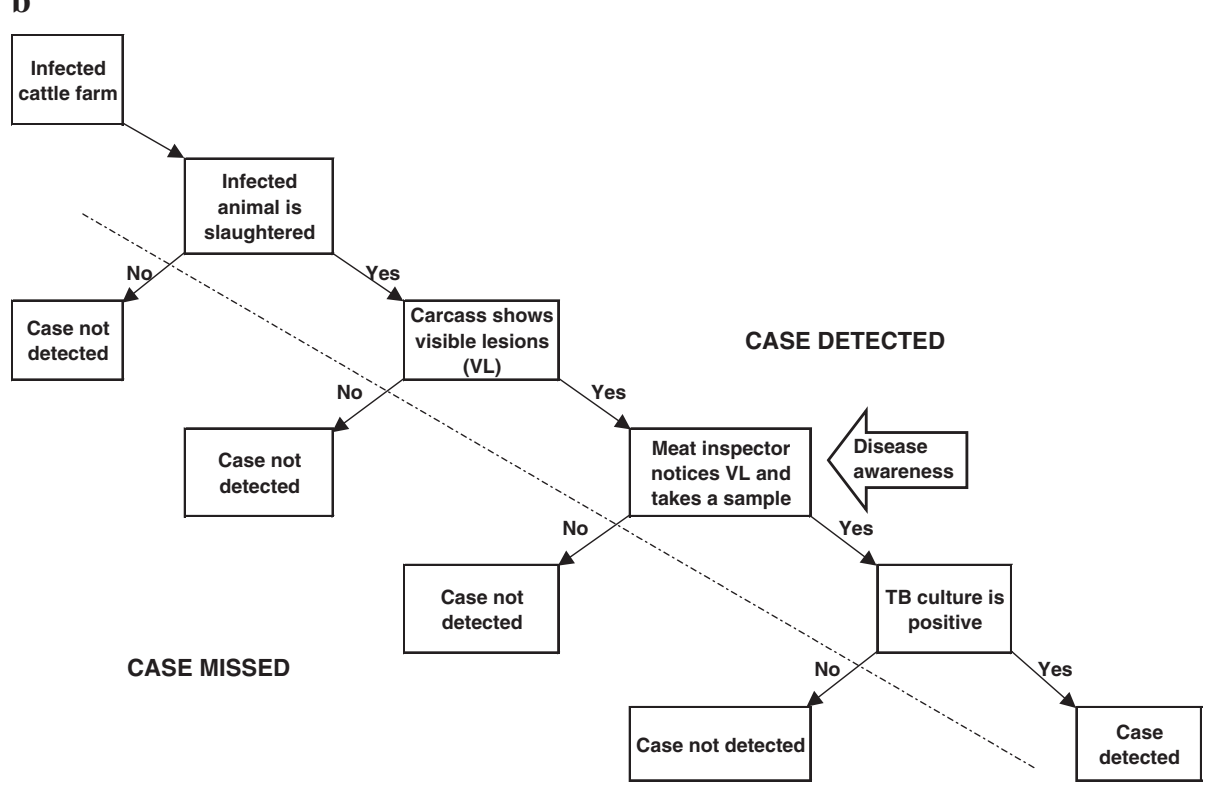

Figure 2. Scenario tree for clinical surveillance of bovine tuberculosis (bTB) on a cattle farm (a), and slaughterhouse surveillance of bTB through meat inspectors (b). 


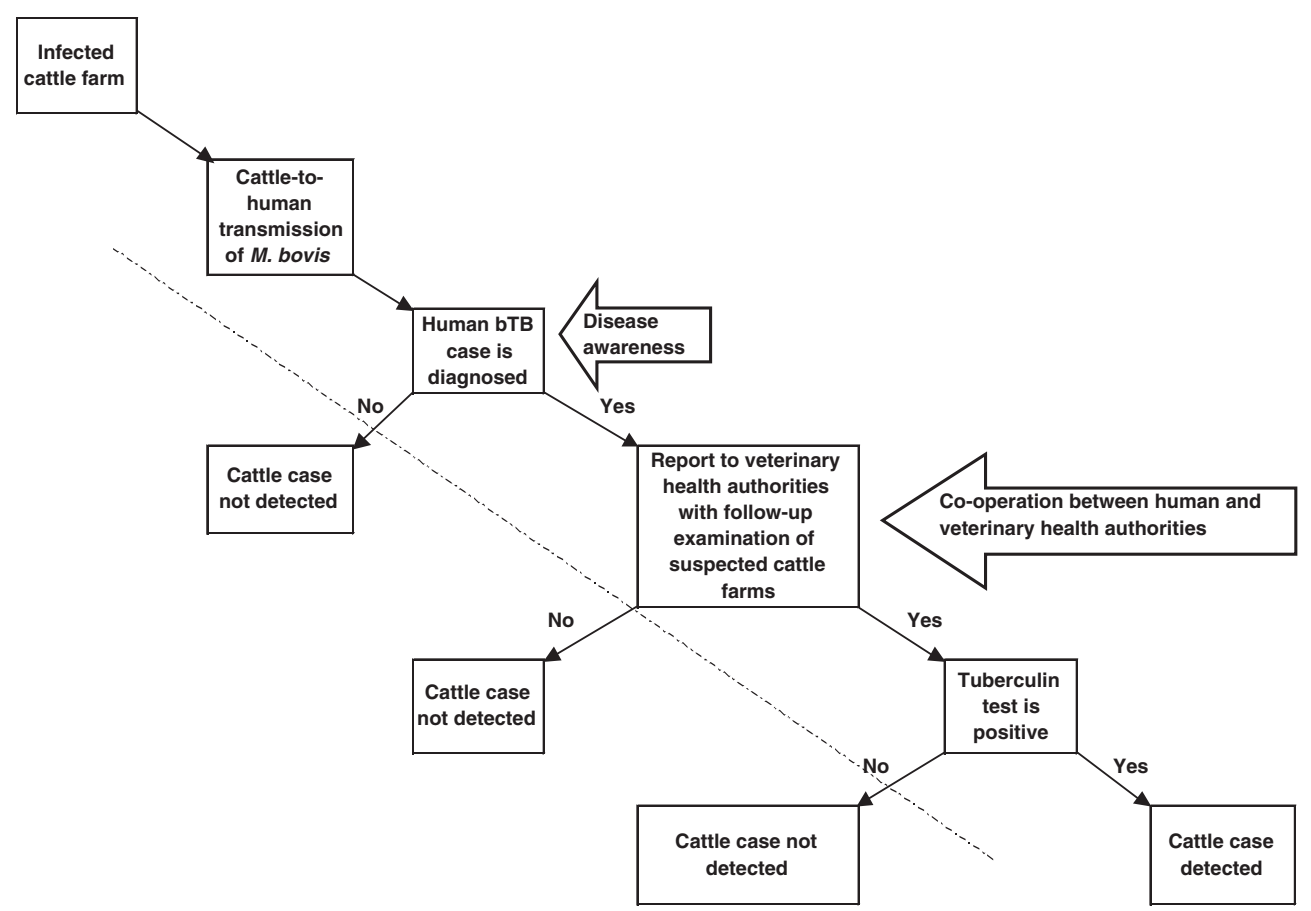

Figure 3. Scenario tree for the indirect surveillance of bovine tuberculosis (bTB) in cattle through detection of human cases.

Table I. Qualitative description of disease awareness categories and probabilities used in a simulation to assess passive surveillance of bovine tuberculosis in Switzerland.

\begin{tabular}{|c|c|c|c|c|c|}
\hline \multirow[t]{2}{*}{ Category } & \multirow{2}{*}{$\begin{array}{l}\text { Median } \\
\text { value }\end{array}$} & \multirow{2}{*}{$\begin{array}{c}\text { Distribution } \\
\text { (@Risk function) }\end{array}$} & \multicolumn{3}{|c|}{ Distribution parameters } \\
\hline & & & $\begin{array}{l}\text { Minimum } \\
\text { value }\end{array}$ & $\begin{array}{l}\text { Most likely } \\
\text { value }\end{array}$ & $\begin{array}{c}\text { Maximum } \\
\text { value }\end{array}$ \\
\hline Low disease awareness (L) & 0.20 & RiskPert & 0.10 & 0.20 & 0.30 \\
\hline Medium disease awareness (M) & 0.50 & RiskPert & 0.40 & 0.50 & 0.60 \\
\hline High disease awareness $(\mathrm{H})$ & 0.80 & RiskPert & 0.70 & 0.80 & 0.90 \\
\hline Very high disease awareness (VH) & 1.00 & Fixed value & 1.00 & 1.00 & 1.00 \\
\hline
\end{tabular}

Table II. Levels of disease awareness for each passive surveillance system component (SSC) considered for the passive surveillance of bovine tuberculosis in Switzerland. Scenario 1 represents the assumed current disease awareness levels in Switzerland and scenarios 2-4 are hypothetical.

\begin{tabular}{llcccc}
\hline SSC & Description of disease awareness & \multicolumn{4}{c}{ Level of disease awareness } \\
\cline { 3 - 5 } & & Scenario 1 & Scenario 2 & Scenario 3 & Scenario 4 \\
\hline CLIN-SSC & Disease awareness of farmer & $\mathrm{M}$ & $\mathrm{H}$ & $\mathrm{VH}$ & VH \\
& Disease awareness of practitioner & $\mathrm{L}$ & $\mathrm{M}$ & $\mathrm{H}$ & VH \\
SLI-SSC & Disease awareness of meat inspector & $\mathrm{M}$ & $\mathrm{H}$ & $\mathrm{VH}$ & VH \\
HS-SSC & Cooperation between human and & $\mathrm{L}$ & $\mathrm{M}$ & $\mathrm{H}$ & VH \\
& animal health authorities & & & &
\end{tabular}

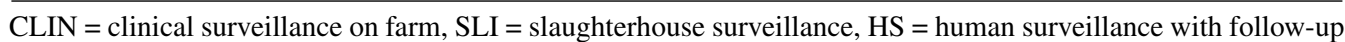
investigations of suspected cattle farm, $\mathrm{L}=$ low, $\mathrm{M}=$ medium, $\mathrm{H}=$ high, $\mathrm{VH}=$ very high. 
Table III. Input parameters used in a stochastic scenario tree model to assess the surveillance for bovine tuberculosis in Switzerland.

\begin{tabular}{lcc}
\hline Description of input parameter & Value & Source \\
\hline $\begin{array}{l}\text { Design prevalence for disease } \\
\text { freedom }\left(\mathrm{P}^{*} \mathrm{H}\right)\end{array}$ & 0.002 & OIE Animal Health Code \\
\end{tabular}

Within-herd prevalence $\left(\mathrm{P}^{*} \mathrm{~A}\right)$ (assumption of 1 infected animal/herd)

1/(RiskCumul Herd size)

Clinical surveillance on farm:

Probability that infected cattle show clinical signs (weakness, coughing, loss of weight)

Probability that farmer calls practitioner

Probability that practitioner conducts

tuberculin test

Sensitivity of single intradermal tuberculin test (SITT)

Slaughterhouse surveillance:

Probability that animal is

slaughtered

Probability that lesions are visible in infected cattle

Probability that samples are taken

post mortem by meat inspector

Sensitivity of bacteriological culture

Human surveillance:

Probability that infected person is detected

Probability for a report to the State Veterinary Service with follow-up examination of suspect cattle farm(s)

$\left.\begin{array}{c}\text { RiskPert } \\ (0.0001 ; 0.0005 ; \\ 0.001) \\ \text { RiskPert } \\ (0.40 ; 0.50 ; 0.60) \\ \text { RiskPert } \\ (0.15 ; 0.25 ; 0.35) \\ \text { RiskPert } \\ (0.77 ; 0.86 ; 0.95)\end{array}\right\}$

RiskPert

$(0.20 ; 0.22 ; 0.25)$

RiskPert

$(0.09 ; 0.30 ; 0.37)$

RiskPert

$(0.40 ; 0.50 ; 0.60)$

RiskPert

$(0.88 ; 0.90 ; 0.912)$
RiskPert

$(0.90 ; 0.95 ; 0.99)$

RiskPert

$(0.15 ; 0.25 ; 0.35)$
${ }^{1}$ The qualitative information of the experts was turned into quantitative consensus estimate according to the categories provided in Table I.

1997 using the single intradermal tuberculin test (SITT) and whole herd testing. Because tuberculin testing is very time-consuming and expensive and a very large sample size is required to substantiate freedom from disease by RS, a more cost-effective approach was warranted such as TS of herds in high-risk strata. Therefore, RS with a sample size of 1000 herds was compared to a TS of 500 herds
Agrarinformationssystem

Schweiz (2006)

Expert opinion (based on specialists from UK)

Personal opinion $^{1}$

[3]

Personal communication,

H. Schwermer, FVO

Expert opinion (meat inspectors of the nine largest Swiss slaughterhouses) ${ }^{1}$

[4], pers. opinion discussion with 2 bTB

[5], [8]

Expert opinion (two Swiss public health specialists) ${ }^{1}$ in high-risk stratum 'wildlife' and also to a more specified targeted sampling of 50 cattle farms belonging to both the high-risk stratum 'wildlife

The expected herd prevalence $(\mathrm{P} * \mathrm{H})$ was set to $0.2 \%$ and within-herd prevalence $(\mathrm{P} * \mathrm{~A})$ was modeled using true herd size distribution and a within-herd prevalence of one infected animal. and trade'. 
Table IV. Median sensitivities (CSe) of the passive surveillance system components (SSC) considered for the surveillance of bovine tuberculosis in Switzerland: clinical surveillance on farm (CLIN-CSe), slaughterhouse surveillance (SLI-CSe) and human surveillance (HS-CSe). Comparison of four different scenarios (see Tab. II) with increasing disease awareness. The two figures in brackets indicate the corresponding $5 \%$ tile and $95 \%$ tile values, respectively.

\begin{tabular}{lccc}
\hline Scenario & \multicolumn{3}{c}{ SSC sensitivities } \\
\cline { 2 - 4 } & CLIN-CSe & SLI-CSe & HS-CSe \\
\hline 1 & 0.006 & 0.556 & $2.99 \mathrm{E}-10$ \\
& $(0.002-0.01)$ & $(0.397-0.727)$ & $(1.05 \mathrm{E}-10-5.68 \mathrm{E}-10)$ \\
2 & 0.023 & 0.728 & $8.05 \mathrm{E}-10$ \\
& $(0.011-0.037)$ & $(0.559-0.871)$ & $(2.82 \mathrm{E}-10-1.30 \mathrm{E}-09)$ \\
3 & 0.045 & 0.804 & $1.33 \mathrm{E}-09$ \\
& $(0.022-0.071)$ & $(0.643-0.922)$ & $(4.56 \mathrm{E}-10-2.04 \mathrm{E}-09)$ \\
4 & 0.056 & 0.804 & $1.69 \mathrm{E}-09$ \\
& $(0.027-0.087)$ & $(0.643-0.922)$ & $(5.75 \mathrm{E}-10-2.49 \mathrm{E}-09)$ \\
\hline
\end{tabular}

\section{RESULTS}

The results for the passive SSC showed that CSe, i.e. the probability to detect at least one positive case over one year was very low for CLIN and HS (Tab. IV). CSe was increased when disease awareness was improved (scenarios 2-4), but the gain was limited for CLIN due to the lack of pathognomonical clinical signs and for HS due to the low incidence of $0.036 / 100000$ human bTB cases per year in Switzerland. The median SLI-CSe in Switzerland was 55.6\% given a medium disease awareness in meat inspectors. The sensitivity analysis showed that the most influential factor for SLI-CSe was the probability that bTB lesions are visible in infected cattle. This biological factor cannot be influenced by management. But the disease awareness of meat inspectors and therefore the probability that visible lesions will be detected if present is influenceable. If the disease awareness of meat inspectors was assumed to be high or very high, SLI-CSe was increased to $72.8 \%$ and $80.4 \%$, respectively, indicating a potential benefit from awareness campaigns (Tab. IV).

The results for the active SSC (Tab. V) clearly showed that TS in high-risk stratum 'wildlife' was more sensitive, with a CSe of $96.7 \%$, and with half the sample size ( $n=500)$, when compared with RS, which had a CSe of $82.1 \%$ from a sample size of 1000 herds. CSe for the even more targeted sampling in the risk stratum 'wildlife and trade' was still $95.8 \%$ with a sample size of just 50 herds.

According to the sensitivity analysis, RR for the stratum 'wildlife' was the most influential parameter for TS in both 'wildlife' and 'wildlife and trade' strata.

Assuming that the passive SSC (CLIN, SLI and HS) and the active SSC (RS or TS) are independent we used Equation (3) to calculate SSe for bTB in Switzerland.

The probability to find at least one infected cattle herd in Switzerland using solely passive surveillance (CLIN, SLI and HS) and its assumed performance was calculated to have a median value of $55.8 \%$ with 5th and 95th percentiles of $40.1 \%$ and $72.6 \%$, respectively, provided that bTB was present at a prevalence of $\geq 0.2 \%$. If disease awareness in all persons involved in the reporting chain was raised to 'very high', the overall passive SSe was increased to $81.5 \%(0.661-0.924)$. The combination of passive SSC with a RS of 1000 farms resulted in a median SSe of $92.1 \%$ (Tab. V). Conducting a TS of only 500 herds in 'wildlife' stratum or an even more targeted sampling of 50 herds in 'wildlife and trade' stratum produced a higher median SSe of $98.6 \%$ and $98.2 \%$, respectively, with $50 \%$ and $5 \%$ of the initial sample size, respectively. 
Table V. Comparison of the surveillance system component sensitivity of random sampling (RS) and targeted sampling (TS) in high-risk strata for bovine tuberculosis in Switzerland. Stratum 'wildlife' = farms with contact to wildlife reservoirs, stratum 'trade' = farms with a high number of animal movements. Resulting sensitivities are provided for RS and TS individually as well as the combined sensitivities of RS and TS with passive surveillance system components (SSC) for scenario 1 (see Tab. II).

\begin{tabular}{lcccr}
\hline SSC & $\begin{array}{c}\text { Sample } \\
\text { size }\end{array}$ & \multicolumn{3}{c}{$\begin{array}{c}\text { Percentiles of surveillance } \\
\text { system sensitivity }\end{array}$} \\
\cline { 3 - 5 } & & 5 th & 50 th & 95 th \\
\hline RS & 1000 & 0.800 & 0.821 & 0.840 \\
TS 'wildlife' & 500 & 0.959 & 0.967 & 0.974 \\
TS 'wildlife and trade' & 50 & 0.948 & 0.958 & 0.966 \\
Passive SSC combined with RS & 1000 & 0.890 & 0.921 & 0.952 \\
Passive SSC combined with TS 'wildlife' & 500 & 0.979 & 0.986 & 0.992 \\
Passive SSC combined with TS 'wildlife and trade' & 50 & 0.973 & 0.982 & 0.989 \\
\hline
\end{tabular}

Therefore, TS appeared to be a cost-effective strategy to increase SSe of bTB in Switzerland in a situation of very low prevalence as expected in a situation where the infection would be re-emerging.

\section{DISCUSSION}

Using scenario tree modelling for the evaluation and optimisation of surveillance systems for rare and emerging diseases provides an essential tool for the planning of efficient surveillance strategies. Based on this methodology, the sensitivities of active as well as passive surveillance activities can be quantified and combined to estimate overall surveillance system sensitivity. Our results showed that structured assessment of the quality of surveillance system components can identify strengths and weaknesses of existing surveillance activities and help identify approaches where changes are likely to be more costeffective. In a situation of generally decreasing resources, rigorous evaluation of ongoing surveillance activities is warranted in order to assure optimal use of personnel and finances.

The key hurdle in using a quantitative approach for evaluating surveillance system performance is the lack of sufficient, available data. In such a situation expert opinion may be used [13].

Clearly, the outcome of such a stochastic scenario tree model is only as good as the input parameters are valid. But while expert opinion will remain subjective to some extent, it allows obtaining a general impression and insight into the relative contributions of SSC, and into effects of such measures as increasing disease awareness through campaigns as leaflets, talks or articles in professional journals. It is also possible to simulate and analyse the effect of varying input parameters on the SSC and to directly explore the effects of several changeable parameters on the sensitivity of a surveillance system. Such knowledge may help make decisions about prioritisation of measures and where to allocate human and financial resources. Another advantage of scenario tree models is that they can easily be updated and re-run once new data or findings become available.

The bTB example illustrates that disease awareness and reporting of suspect cases plays a crucial role in passive surveillance activities. But the probability that suspect cases are reported depends not only on farmers' and veterinarians' knowledge about a certain disease, but also on other factors like compensation payment for culled animals and economical drawbacks for suspect cases, and it is therefore difficult to quantify. In the absence of data, the classification of disease awareness remains subjective to some extent and there is a need for further investigation of most likely values. Although this highlights an important knowledge gap which should be addressed, we believe that the relative impact of disease awareness on the sensitivity of

Page 10 of 12 (page number not for citation purpose) 
passive surveillance as shown by our results remains valid.

While the level of disease awareness may be increased by information and campaigns, sustaining such disease awareness may be difficult, and this can affect the sensitivity of a surveillance system over time. We hypothesize that disease awareness is dependent on the disease situation; i.e. disease awareness is more difficult to increase and keep high for rare diseases compared to endemic disease situations. It is also expected that disease awareness will be variable over time, e.g. higher right after an information campaign or a recent outbreak. Because a sustainable augmentation in disease awareness is difficult to achieve and maintain over time in a disease free situation, it may be necessary to conduct supplementary surveys.

In the example of bTB surveillance, tracing back of human cases (HS) as a possible information source for the presence of bTB in cattle population was of negligible value because of the disease free situation in Switzerland. But this SSC has the potential to be useful in endemic situations. The most influencing parameters of this SSC are the disease awareness of human medical professionals followed by the probability for reporting human bTB cases to the State Veterinary Service and the follow-up examination of suspected cattle farms. This supports the idea of 'One Medicine' [18].

The most critical factor when assessing the relative sensitivity of different survey designs is the relative risk or increased probability of occurrence of infection in the high-risk strata. In the bTB example, risk factors and their relative risks were integrated in the scenario tree approach, and it was shown that TS in high-risk strata was much more effective than RS with regard to sample size. Knowledge about the factors influencing the probability of infection for a specific disease is crucial in order to identify the high-risk strata and to conduct a TS in those highrisk strata. If a TS is conducted in a stratum misclassified as high-risk, the evidence of the survey is invalid. In order to obtain a robust basis for decision making, published data obtained from epidemiological studies are required. However, these may be expensive and not practically feasible in the case of an emerging infection. But when resources are severely limited, targeted efforts may be more beneficial even on the basis of qualitative risk assessments and expert opinion. Such an approach can offer a structured tool to priority setting for authorities in low-income countries.

In order to decide if it is more costefficient to increase disease awareness or to conduct a survey, the direct costs for an increase in disease awareness (campaigns, training programmes for animal caretakers and veterinarians) and the indirect costs of a higher disease awareness (more suspect cases with costs for practitioners to take samples and laboratory investigations, economical losses due to eventual animal trade bans in case of suspects) must be quantified and compared to the costs for a survey. These economic considerations need further attention.

Acknowledgements. We thank Martin Vordermeier and Andy Mitchell from Veterinary Laboratories Agency, UK, for their helpful comments on bTB input parameters. We thank Jakob Zinsstag from Swiss Tropical Institute, Basel and Christian Griot from Institute of Virology and Immunoprophylaxis, Mittelhäusern Switzerland for their support and constructive discussions related to this project. We thank Heinzpeter Schwermer, Federal Veterinary Office, for his help modelling the Swiss cattle population structure, Michael Binggeli for making the map in Figure 1 and Isabel Reding for her helpful comments on the mathematical equations. Work leading to this publication was funded by the National Science Foundation (Grant No. 3200B0109592.1).

\section{REFERENCES}

[1] Bruhn S., Andere Zoonosen, in Schweizer Zoonosebericht 2006, BVET Magazin (2007) 3:40.

[2] Corner L., Melville L., McCubbin K., Small K.J., McCormick B.S., Wood P.R., Rothel J.S., Efficiency of inspection procedures for the detection of tuberculous lesions in cattle, Aust. Vet. J. (1990) 67:389-392.

[3] Cousins D.V., Mycobacterium bovis infection and control in domestic livestock, Rev. Off. Int. Epizoot. (2001) 20:71-85. 
[4] Cousins D.V., Florisson N., A review of tests available for use in the diagnosis of tuberculosis in non-bovine species, Rev. Off. Int. Epizoot. (2005) 24:1039-1059.

[5] De la Rua Domenech R., Bovine tuberculosis in the European Union and other countries: current status, control programmes and constrains to eradication, Gov. Vet. J. (2006) 16:19-45.

[6] Doherr M.G., Audigé L., Monitoring and surveillance for rare health-related events: a review from the veterinary perspective, Philos. Trans. R. Soc. Lond. B Biol. Sci. (2001) 356:1097-1106.

[7] Dondo A., Ferroglio E., Goria M., Moda G., Ruocco L., Vignetta P., Economic significance of bovine tuberculosis in Italy and effect of M. bovis infection in wild swine, in: Thoen C.O., Steele J.H., Gilsdorf M.J. (Eds.), Mycobacterium bovis infection in animals and humans, Blackwell Publishing, Ames, Iowa, USA, 2006, pp. 117-124.

[8] Fritsche A., Engel R., Buhl D., Zellweger J.P., Mycobacterium bovis tuberculosis: from animal to man and back, Int. J. Tuberc. Lung Dis. (2004) 8: 903-904.

[9] Grange J.M., Mycobacterium bovis infection in human beings, Tuberculosis (Edinb.) (2001) 81:71-77.

[10] Johnston W.T., Gettinby G., Cox D.R., Donnelly C.A., Bourne J., Clifton-Hadley R., et al., Herd-level risk factors associated with tuberculosis breakdowns among cattle herds in England before the 2001 foot-and-mouth disease epidemic, Biol. Lett. (2005) 1:53-56
[11] Kaneene J.B., Miller R., Meyer R.M., Abattoir surveillance: the US experience, Vet. Microbiol. (2006) 112:273-282.

[12] Lilienfeld D.E., Stolley P.D., Foundations of epidemiology, 3rd ed., Oxford University Press, Oxford, UK, 1994.

[13] Martin P.A.J., Cameron A.R., Greiner M., Demonstrating freedom from disease using multiple complex data sources 1: A new methodology based on scenario trees, Prev. Vet. Med. (2007) 79: 71-97.

[14] Pavlik I., The experience of new European Union Member States concerning the control of bovine tuberculosis, Vet. Microbiol. (2006) 112: 221-230.

[15] Slingenbergh J., Gilbert M., de Balogh K., Wint W., Ecological sources of zoonotic diseases, Rev. Off. Int. Epizoot. (2004) 23:467-484.

[16] Stärk K.D.C., Regula G., Hernandez J., Knopf L., Fuchs K., Morris R.S., Davies P., Concepts for risk-based surveillance in the field of veterinary medicine and veterinary public health: Review of current approaches, BMC Health Serv. Res. (2006) 6:20.

[17] Thoen C., LoBue P., de Kantor I., The importance of Mycobacterium bovis as a zoonosis, Vet. Microbiol. (2006) 112:339-345.

[18] Zinsstag J., Schelling E., Wyss K., Mahamat M.B., Potential of cooperation between human and animal health to strengthen health system, Lancet (2005) 366:2142-2145. 